\title{
Síndrome de Burnout en docentes universitarios
}

\section{Burnout syndrome in university teachers}

César Augusto Chávez Orozco

Universidad Internacional del Ecuador, Ecuador

Autor para correspondencia: cesarchavez966@ hotmail.com

Fecha de recepción: 30 de Agosto de 2016 - Fecha de aceptación: 28 de Septiembre de 2016

Resumen: Los procesos organizativos, han sufrido grandes cambios, debido a la competitividad impuesta por las presiones económicas derivadas de la industrialización, automatización, globalización y otros fenómenos económicos, políticos y sociales, que repercuten directamente en la salud del trabajador, al no poder adaptarse a las nuevas reglas impuestas por el mercado laboral. Ante esta situación, ha cobrado importancia, el llamado Síndrome de Burnout o de desgaste profesional, que se manifiesta con cambios psicológicos que van incrementando su intensidad desde el descontento y la irritabilidad hasta estallidos emocionales, especialmente en profesionales en relación de dependencia, fundamentalmente en el área de prestación de servicios (docentes, abogados, policías, médicos, enfermeras, etc.). Esta situación se agrava, si el individuo ejecuta sus labores en condiciones inapropiadas, con turnos excesivos, inseguridad en el cargo, remuneración insuficiente y carencia de recursos materiales o humanos indispensables para una correcta ejecución de sus tareas. El presente estudio pretende analizar la presencia del síndrome de burnout en docentes universitarios en Latinoamérica y España, como un elemento importante en el bienestar y en la calidad de vida laboral del docente universitario; a través de realizar una revisión sistemática de la literatura científica publicada en el periodo 2005 - 2015. Se realizó una búsqueda bibliográfica en bases de datos (ebsco, e-book, redalyc, scielo, Dialnet, google académico), considerando países latinoamericanos y España. Se examinaron todos los artículos existentes hasta el 30 de Octubre de 2015 y se definieron los criterios que aseguran la compatibilidad entre los estudios. Se encontraron 152 artículos de los cuales 86 eran de estudios empíricos y 66 de revisiones bibliográficas. De los artículos de estudios empíricos (86), quedaron definitivamente 35 documentos, dando una muestra poblacional de 6842 docentes universitarios. La mayor parte de los autores utilizó el Maslash Burnout Inventory (MBI), como instrumento para la evaluación del síndrome de Burnout.

Palabras claves: burnout; psicopatologías; estrés Abstract: Currently, the world has imposed a dizzying pace in all areas of our lives, one of them, the labor development of the individual. Organizational processes, have undergone major changes, due to the competitiveness imposed by economic pressures of industrialization, automation, globalization and other economic, political and social phenomena that directly affect the health of workers, unable to adapt to the new rules imposed by the labor market. In this situation, it has become important, the so-called burnout syndrome or burnout, which manifests with psychological changes that are increasing in intensity from discontent and irritability to emotional outbursts, especially professionals as employees, mainly in the service delivery area (teachers, lawyers, police). This situation is exacerbated if the individual performs his duties in inappropriate 
conditions, with excessive shifts, insecure tenure, inadequate remuneration and lack of material and human resources necessary for proper execution of their tasks. Burnout has become the most researched psychological effects work in recent decades, demonstrating the growing number of publications that address. All this has led specialists to rule in favor of the inclusion of this syndrome as an occupational disease, with all the legal and economic consequences that entails. It is important to assess the state of the art of this phenomenon and to place in perspective the problem has reached dimension. The present study aims to analyze the presence of burnout syndrome among university teachers in Latin America and Spain, as an important element in the welfare and quality of working life of university teachers; through a systematic review of the scientific literature published in the period 2005-2015. A literature search was performed in databases (EBSCO, e-book, redalyc, scielo, Dialnet, academic google), considering Latin American countries and Spain. All articles that examined until October 30, 2015 and criteria to ensure compatibility between studies were defined. 152 articles of which 86 were empirical studies and 66 literature reviews found. Articles of empirical studies (86), were definitely 35 documents, givinga population sample of6842university teachers. Most of the authors used the MBI instrument, there is a prevalence of burnout in women than in men.

Key words: burnout; psychopathology; stress

\section{Introducción}

El mundo actual, ha impuesto un ritmo acelerado en todos los ámbitos de la vida del ser humano, siendo uno de ellos, el desenvolvimiento en el ámbito laboral, impuesto por la competitividad, las presiones económicas, la globalización. Estas han determinado grandes cambios en la organización del trabajo, afectando directamente la salud física, mental y social del individuo, al no poder adaptarse a estas nuevas reglas impuestas por el mercado laboral (Castro, 2005).

El diccionario de la Real Academia Española, define al trabajo como la acción y efecto de trabajar, y trabajar, como ocuparse en cualquier actividad física o intelectual (Diccionario REA). El trabajo tiene dos características fundamentales: la tecnificación y la organización. Por tecnificación, se entiende las máquinas y herramientas que inventa el individuo para llevar a cabo la transformación del medio ambiente de forma cada vez más cómoda; la organización de trabajo, se relaciona con la asignación de tareas determinadas a individuos concretos para conseguir el mismo resultado con menos esfuerzo que al hacer individualmente (Bestatén, 2008).

El trabajo si bien representa una actividad beneficiosa para la economía del trabajador y para su salud mental individual y colectiva, ha estado inmerso en un mundo de cambios constantes, productos de la industrialización, automatización, globalización y otros fenómenos económicos, políticos y sociales, que generan para la población trabajadora daño biológico, social o psicológico, tales como frustración, insatisfacción y estrés en los lugares de trabajo, que producen diferentes reacciones en los trabajadores; esta situación los va conduciendo a una forma de problemas de salud, como trastornos del comportamiento y enfermedades psicosomáticas, que surgen como consecuencia de manejar incorrectamente el efecto de estos factores y entre las que se pueden mencionar: al Mobbing, Karochi y el Burnout. 
Los contextos socio-económicos turbulentos, producidos a finales del siglo XX y principios del XXI se han caracterizado por el aumento de los estresores psicológicos en el trabajo, como los denominados estresores de rol, que se han destacado por su impacto en la satisfacción de los trabajadores, favoreciendo la experiencia del Burnout o síndrome de quemarse en el trabajo (SQT). Es importante mencionar que, los roles tienen la función de reducir la incertidumbre en el puesto de trabajo, pero pueden ser origen de problemas cuando aparece el conflicto, la sobrecarga o la ambigüedad de rol (Orgambídez Alejandro, 2015).

El sector universitario a nivel mundial, ha sufrido cambios importantes durante las pasadas dos décadas, como son: la caída de salarios, el incremento de posiciones académicas cada vez más inestables, la presión y carga de trabajo debida a la necesidad de atraer fondos de financiamiento externos, convirtiéndolo en fuente de estrés (Avargues, 2010).

En Ecuador, desde el año 2008, también se han producido cambios importantes en la nueva regulación para la carrera y escalafón de las plantas académicas del sistema de educación superior ecuatoriano (Minteguiaga, 2013), estos cambios, se producen como resultado de políticas de estado, de transformaciones sociales, culturales; consecuencia a su vez, de la globalización, la sociedad del conocimiento y la fuerte presencia de tecnologías en nuestras vidas.

\section{El estrés laboral}

El estrés laboral, es la reacción que puede tener el individuo ante exigencias y presiones laborales que no se ajustan a sus conocimientos y capacidades y que ponen a prueba su capacidad para afrontar la situación; puede producirse en situaciones laborales muy diversas y se agrava cuando el trabajador siente que no recibe suficiente apoyo de sus supervisores y colegas, y cuando tiene un control limitado sobre su trabajo o la forma en que puede hacer frente a las exigencias y presiones laborales (Leka, 2004).

Existen tres orientaciones sobre el estrés, que han marcado el desarrollo de la teoría general del estrés y de su aplicación al campo psicopatológico, que son: el estrés focalizado en la respuesta, propuesta por Selye, el estrés centrado en el estímulo, formulado por Holmes y Rahe, y el estrés basado en la valoración cognitiva, planteado por Lazarus(Berrio, 2011).

Teoría basada en la respuesta.- Según esta teoría, el estrés se desdobla en eustrés (o eutrés) y distrés. El eustrés es la respuesta positiva de adaptación ante las situaciones de amenaza; y el distrés, es el fracaso a esta adaptación que se manifiesta en el individuo en sentimientos negativos, (Figura 1). Según este enfoque, adquiere relevancia los conceptos de estresor, como aquello que produce reacción, y respuesta, cómo se manifiesta; y la posibilidad de considerar al estrés como motivador (eustrés) o como demanda excesiva (distrés) (Zabala, 2008), (Berrio, 2011).

Para Selye (citado en Berrio, 2011), el estrés se concibe como una respuesta no específica del organismo, ante las demandas que se hacen. Para este autor, el estrés es "el estado que se manifiesta por un síndrome específico, consistente en todos los cambios inespecíficos inducidos dentro de un sistema biológico". El estrés no tiene una causa particular, el estresor, es un factor 
que afecta la homeostasis del organismo, y puede ser un estímulo físico, psicológico, cognitivo o emocional (Berrio, 2011).

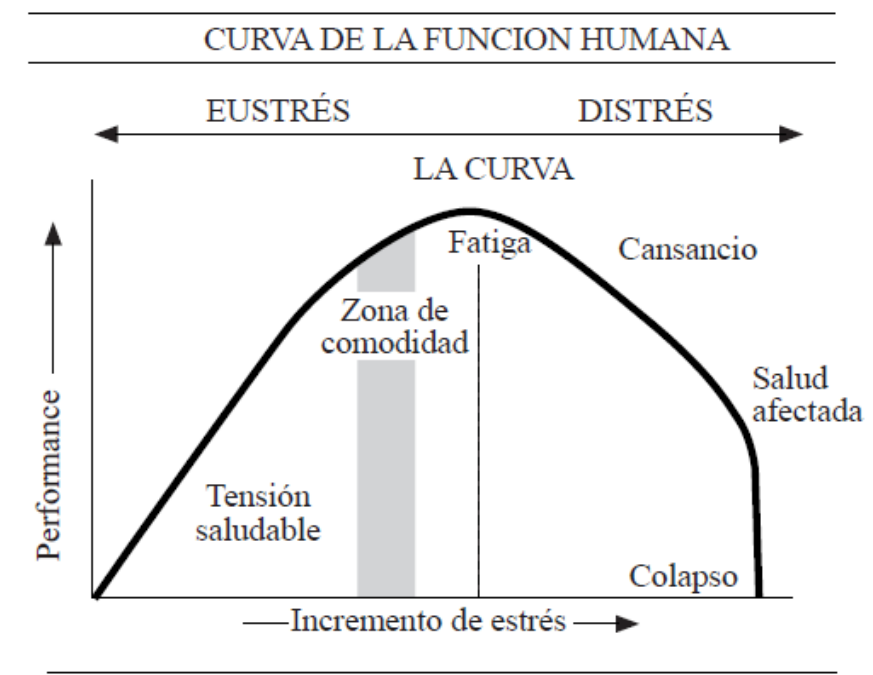

Figura 1. La curva de la función humana Adaptado de: Nixon, P:Practioner, 1979

Diagrama del American Institute of Stress (AIS s/a, trad. libre)

Fuente: (Zabala, 2008)

Teoría basada en el estímulo.-Esta teoría, categoriza a los estresores como psicosociales y biológicos. Los estresores psicosociales son estímulos ambientales externos y dependen de la interpretación cognitiva del sujeto para que se conviertan en estrés, como ejemplos se puede citar al ruido y otros tipos de contaminación o condiciones de un medio físico. Los estresores biológicos, son aquellos que producen cambios bioquímicos o eléctricos en el organismo, como por ejemplo, el consumo sociocultural de café y tabaco. (Zabala, 2008), (Berrio, 2011)

Teorías interaccionales o transaccionales.- Son las teorías más completas, y conceptualizan a los estresores como demandas hechas por el ambiente interno o externo que afectan el balance de un individuo y el bienestar físico o psicológico, y requieren de una acción para restablecerlo (Zabala, 2008), (Berrio, 2011).

\section{Psicopatologías Laborales}

Se define a la psicopatología del trabajo como "el análisis de sufrimiento psíquico resultante de la confrontación de los hombres con la organización del trabajo"; también se puede decir que es "el análisis psicodinámico de los procesos intersubjetivos movilizados por la situación de trabajo" (Dejours, 1998).La psicopatología Laboral estudia los problemas y riesgos psicológicos generados en el trabajo como el estrés, el burnout, el acoso psicológico y el acoso laboral.

\section{El burnout y sus dimensiones}

\section{Antecedentes y actualidad}


La docencia es una de las profesiones más afectadas por el síndrome de Burnout. Fue descrito originalmente por Freudenberger (1974) para referirse a las reacciones físicas y mentales que observó en un grupo de personas que trabajaban en una clínica de desintoxicación. Posteriormente, Maslach y Jackson (1981) definieron el burnout como un trastorno adaptativo crónico, asociado a las demandas psicosociales que requiere el trabajo directo con personas, experimentando un desequilibrio prolongado entre las demandas y los recursos de afrontamiento.

El síndrome de burnout, es una de las principales consecuencias del malestar docente, se define como "el conjunto de consecuencias negativas que afectan a la personalidad del profesor a partir de la acción combinada de condiciones psicológicas y sociales en que se ejerce la docencia” (Esteve, 1987) referenciado en (Bermejo \& Prieto, 2005).

El malestar docente es una realidad a la que miles de docentes se enfrentan diariamente, provocando consecuencias como trabajar desmotivado o estresado, convirtiéndose en un grave problema social más que personal; siendo el estrés laboral una de las principales causas de baja (Esteve, 1987).

Cuando se habla de malestar docente, se hace referencia a un fenómeno que afecta a una buena parte del profesorado y que se origina en respuesta a una serie de factores o acontecimientos negativos que los aquejan en el marco educativo. Tal respuesta está precedida de un estilo atributivo pesimista que sería el responsable del incremento de los índices de estrés laboral ("burnout") y ansiedad que exhiben los docentes afectados, pudiéndose llegar a alcanzar la depresión como resultado último (De la Torre, 2006).

El estrés se ha convertido en una patología que acompaña a gran parte de la población trabajadora. El síndrome de Burnout, también llamado síndrome de estar quemado o de desgaste profesional, se considera como la fase avanzada del estrés laboral, como consecuencia del estrés laboral crónico. El Burnout, se produce especialmente en organizaciones de servicios y afecta a profesionales que tratan con clientes o usuarios, como docentes, médicos, enfermeras, policías, etc.

\section{Importancia del tema}

El estudio del síndrome de Burnout o quemarse por el trabajo, está unido a la necesidad de estudiar los procesos de estrés laboral, así como al reciente hincapié que las instituciones han hecho sobre la necesidad de preocuparse más de la calidad de vida laboral que ofrecen a sus empleados. Actualmente, resulta necesario considerar los aspectos de bienestar y salud laboral a la hora de evaluarla eficacia de una determinada organización, pues la calidad de vida laboral y el estado de salud física y mental que conlleva tiene repercusiones sobre la institución educativa, provocando: ausentismo, rotación, disminución de la productividad, disminución de la calidad, etc.

El síndrome de Burnout afecta a la calidad de vida laboral y el estado de salud física y mental de los docentes, provocando agotamiento emocional en el trabajo, la adopción de actitudes negativas y cínicas hacia los estudiantes y la institución; así como el descontento, la desmotivación y la insatisfacción personal; aspectos que conlleva a repercusiones sobre la 
institución como: ausentismo, rotación, disminución de la productividad, disminución de la calidad de la educación, y otros. Después de un año, muchos de ellos se sentían agotados, irritables, y habían desarrollado una actitud cínica hacia sus pacientes y una tendencia a evitarlos (Sandín, 2008).

El estrés afecta a todas las actividades laborales, sin embrago, afecta a aquellas actividades relacionadas con la prestación de servicios como la educación, servicios sanitarios y el ámbito asistencial; el mismo que cuando llega a niveles crónicos (estrés laboral crónico) puede dar origen a la aparición del síndrome de Burnout o de estar quemado por el trabajo; acompañado además de una serie de síntomas físicos y psicosomáticos (Freudenberger, 1974).

\section{¿Qué es el Burnout?}

El síndrome de Burnout es considerado como una respuesta al estrés laboral crónico producido por la presencia de los Factores Psicosociales Negativos del Trabajo, ya sean propios de la tarea, organizacionales, de interacciones interpersonales, o de otro tipo. Si el Síndrome se padece a lo largo del tiempo, tendrá consecuencias nocivas para el individuo, en forma de enfermedad o falta de salud con alteraciones psicosomáticas, mientras que la organización presentará una baja en la motivación laboral y en el rendimiento de la productividad y calidad de la producción. (Pando Moreno, Aranda Beltran, Aldrete, Flores Salinas, \& Pozos Radillo, 2006, pág. 174)

El síndrome de Burnout se caracteriza por actitudes negativas hacia los sujetos que constituyen el objeto de su trabajo y hacia el propio trabajo, absentismo, uso de alcohol y otras drogas, pesimismo, apatía, motivación laboral escasa o nula, baja implicación laboral, irritabilidad hacia las personas destinatarias del trabajo, preocupación excesiva por el confort y el bienestar laboral, tendencia a racionalizar los fracasos culpando a los usuarios o al sistema, resistencia al cambio y baja creatividad. (Pando Moreno, Aranda Beltran, Aldrete, Flores Salinas, \& Pozos Radillo, 2006, pág. 174)

Freudenberger describe al Burnout como "conjunto de síntomas inespecíficos que pueden aparecer en el ambiente laboral y que son el resultado de una demanda progresiva excesiva". (Castro, 2005, pág. 162)

Maslach y Jackson (1980, 1981, 1985 y 1986), desde una perspectiva psicosocial, afirman que Burnout es un síndrome tridimensional que se desarrolla en profesionales cuyo objeto de trabajo son personas (Guerrero Barona, 2003, pág. 145), ya sean clientes o personal de la empresa, ya sean del mismo u otro nivel jerárquico. Se presenta como consecuencia de la discrepancia entre las expectativas que el individuo deposita en el mundo laboral y una realidad que se muestra frustrante (Castro, 2005, pág. 162). En este fenómeno, intervienen variables emocionales como el agotamiento emocional, variables actitudinales como la despersonalización y variables cognitivo-aptitudinales como la baja realización personal en el trabajo (Marrau, 2004) referido en (Tifner, 2006). Estas tres dimensiones, se describen de la siguiente manera:

Agotamiento emocional.- Cansancio y fatiga que puede manifestarse física, psíquicamente o como una combinación de ambas (Castro, 2005, pág. 163); es la sensación de no 
poder dar más de sí mismo a los demás (Guerrero Barona, 2003). Este agotamiento de los recursos emocionales propios, es debido al contacto con personas destinatarias de esta prestación en condiciones no ajustadas al trabajador los cuales pueden expresar disgusto y hartazgo con su tarea, o de otro modo, sentirse agobiados por la misma.(Tifner, 2006, pág. 281)

Despersonalización.- Se entiende como el desarrollo de sentimientos, actitudes, y respuestas negativas, distantes y frías hacia otras personas, especialmente hacia los beneficiarios del propio trabajo. Se acompaña de un incremento en la irritabilidad y una pérdida de motivación hacia el trabajo. El profesional despersonalizado trata de distanciarse no sólo de las personas destinatarias de su trabajo sino también de los miembros del equipo con los que trabaja, mostrándose cínico, irritable, irónico e incluso utilizando etiquetas despectivas, al mismo tiempo que les atribuye la culpabilidad de sus frustraciones y de su descenso del rendimiento laboral. (Guerrero Barona, 2003), (Castro, 2005, pág. 163),(Tifner, 2006, pág. 281)

Bajo logro profesional y/o personal.- Consistente en un sentimiento de bajo logro o realización profesional y/o personal que surge cuando se verifica que las demandas que se le hace exceden su capacidad para atenderlas de forma competente. Supone respuestas negativas hacia uno mismo y hacia su trabajo, evitación de las relaciones personales y profesionales, bajo rendimiento laboral, incapacidad para soportar la presión y una baja autoestima. La falta de logro personal en el trabajo se caracteriza por una dolorosa desilusión e impotencia para dar sentido al mismo. Se experimentan sentimientos de fracaso personal (falta de competencia, de esfuerzo o conocimientos), carencias de expectativas laborales y una generalizada insatisfacción. Como consecuencia, la impuntualidad, la abundancia de interrupciones, la evitación del trabajo, el absentismo y el abandono de la profesión aparecen como síntomas habituales y típicos de esta patología laboral(Guerrero Barona, 2003)(Tifner, 2006, pág. 281). El bajo logro puede aparecer encubierto y manifestarse por una sensación paradójica de omnipotencia. (Guerrero Barona, 2003, pág. 146)

\section{Factores de riesgo: las causas desencadenantes del burnout}

El síndrome de burnout tiene su origen en el entorno laboral y en las condiciones de trabajo. Adicionalmente, algunas variables de personalidad, sociodemográficas individuales o de entorno personal, pueden provocar evoluciones diferentes en su desarrollo.

Las actuaciones que desencadenan la aparición de este síndrome suelen ser intensas y/o duraderas, porque el burnout se define como un proceso continuo que se manifiesta de una manera paulatina y que va interiorizando el individuo hasta provocar en éste los sentimientos propios del síndrome.

Entre los factores que propician el burnout, aparecen los siguientes:

- El tipo de profesión.

- La variable organizativa.

- El diseño del puesto de trabajo.

- Las relaciones interpersonales.

- La variable individual (características del profesional como edad, sexo, experiencia, rasgos de personalidad). 


\section{El tipo de profesión}

El burnout afecta de modo especial a aquellas profesiones cuyas tareas se concretan en una relación continuada y estrecha con personas: sean clientes o usuarios, sobre todo si entre ambos existe una relación de ayuda y/o de servicio, por ejemplo, personal de salud, educación, administración pública, etc.

Como resumen, el burnout se desarrolla principalmente en ambientes laborales:

- Compuestos por personal que trata directamente con otras personas: clientes, usuarios, alumnos etc....

- Que requieren un alto compromiso laboral, pero que frustran cotidianamente las expectativas profesionales.

- Que someten a fuertes presiones, de aislamiento y menosprecio, a sus empleados, provocando su total desmotivación.

\section{La variable organizativa}

La estructura de la organización y la forma en que se presta el servicio es relevante para identificar problemas de burnout. Los factores más prevalentes pueden ser:

- Estructura de la organización muy jerarquizada y rígida.

- Falta de apoyo instrumental por parte de la organización.

- Exceso de burocracia, "burocracia profesionalizada".

- Falta de participación de los trabajadores.

- Falta de coordinación entre las unidades.

- Falta de formación práctica a los trabajadores en nuevas tecnologías.

- Falta de refuerzo o recompensa.

- Falta de desarrollo profesional.

- Relaciones conflictivas en la organización.

- Estilo de dirección inadecuado.

- Desigualdad percibida en la gestión de los RRHH.

\section{El diseño del puesto de trabajo}

Se convierte en un factor estresante para el trabajador cuando no puede actuar sobre factores como el control del tiempo, la ambigüedad del rol, etc. Los factores negativos relativos al diseño de los puestos son:

- Sobrecarga de trabajo, exigencias emocionales en la interacción con el cliente.

- Descompensación entre responsabilidad y autonomía.

- Falta de tiempo para la atención del usuario (paciente, cliente, subordinado, etc.).

- Disfunciones de rol: conflicto-ambigüedad- sobrecarga de rol.

- Carga emocional excesiva.

- Falta de control de los resultados de la tarea.

- Falta de apoyo social.

- Tareas inacabadas que no tienen fin. 
- Poca autonomía decisional.

- Estresores económicos.

- Insatisfacción en el trabajo.

\section{Las relaciones interpersonales}

Las relaciones con los clientes, usuarios, pacientes etc...., marcan la actividad diaria de las profesiones con mayor incidencia de burnout, generando una serie de factores de riesgo asociados, cómo:

- Trato con usuarios difíciles o problemáticos.

- Relaciones conflictivas con clientes.

- Negativa dinámica de trabajo.

- Relaciones tensas, competitivas, con conflictos entre compañeros y con usuarios.

- Falta de apoyo social.

- Falta de colaboración entre compañeros en tareas complementarias.

- Proceso de contagio social del síndrome de estar quemado.

- Ausencia de reciprocidad en los intercambios sociales.

\section{La variable individual}

Algunas características inherentes al profesional que motivan la aparición del burnout, son:

- Alta motivación para la ayuda.

- Alto grado de empatía.

- Alto grado de altruismo.

- Baja autoestima.

- Constancia en la acción.

- Tendencia a la sobre-implicación emocional.

- Baja autoeficacia.

- Reducidas habilidades sociales

Otros factores a tener en cuenta son, la edad y el sexo:

- La edad

Aunque los estudios no son concluyentes, existe una tendencia a dar relevancia al factor edad, pues el trabajador experimentaría una mayor vulnerabilidad en una etapa de su vida que en otra.

Normalmente, la etapa de mayor riesgo se identifica con los primeros años de carrera profesional, al considerarse el espacio de tiempo más propicio para que se produzca la transición de las expectativas idealistas hacia la práctica cotidiana, aprendiéndose en este tiempo que tanto las recompensas personales, como las profesionales y económicas, no son ni las prometidas ni las esperadas. 
Los estudios realizados, el grupo de edad con mayor cansancio emocional fue el de los mayores de 44 años en quienes también se detectó una falta de realización personal. Esta carencia se encuentra más marcada en los profesionales con mayor antigüedad, aquellos con más de 19 años de ejercicio en la profesión y más de 11 años en el mismo puesto de trabajo. Así, se observa una disminución en la producción y una tendencia a la desorganización que acompañan al agotamiento personal.

- El sexo

La mayor incidencia del estrés laboral en las mujeres, en particular por la doble carga de trabajo que conlleva la práctica profesional y la tarea familiar, podría hacer pensar en que también respecto del burnout tiene una mayor presencia.

\section{Síntomas Del Burnout}

El Burnout presenta diversos síntomas, entre los más habituales se pueden citar los siguientes (Castro, 2005, pág. 164), (Moreno, 2001):

\begin{tabular}{|c|c|c|c|c|c|}
\hline $\begin{array}{l}\text { Psicosomátic } \\
\text { os }\end{array}$ & De conducta & Emocionales & Cognitivos & Sociales & Laborales \\
\hline $\begin{array}{l}\text { - } \begin{array}{l}\text { Fatiga } \\
\text { crónic }\end{array} \\
\text { a. } \\
\text { - } \\
\text { Trast } \\
\text { ornos } \\
\text { del } \\
\text { sueño } \\
\cdot \\
\text { - Ulcer } \\
\text { as. } \\
\text { Desór } \\
\text { denes } \\
\text { gástri } \\
\text { cos, } \\
\text { tensió } \\
\mathrm{n} \\
\text { musc } \\
\text { ular. } \\
\text { Agota } \\
\text { mient } \\
\text { o } \\
\text { físico } \\
\text { y } \\
\text { psíqui } \\
\text { co } \\
\text { que }\end{array}$ & $\begin{array}{ll}\text { - } & \text { Ausentis } \\
\text { mo } & \text { laboral. } \\
\text { - } & \text { Adiccion } \\
\text { es } \\
\text { (tabaco, } \\
\text { alcohol, } \\
\text { drogas, } \\
\text { etc). } \\
\text { Conducta } \\
\text { s } \\
\text { inadaptati } \\
\text { vas. } \\
\text { Desorgan } \\
\text { ización. } \\
\text { Evitación } \\
\text { de } \\
\text { responsa } \\
\text { bilidades. } \\
\text { Evitación } \\
\text { de } \\
\text { decisione } \\
\text { s. }\end{array}$ & $\begin{array}{ll}\text { - } & \begin{array}{l}\text { Irritabili } \\
\text { dad. }\end{array} \\
\text { - } & \begin{array}{l}\text { Incapaci } \\
\text { dad de } \\
\text { concent } \\
\text { ración. }\end{array} \\
\text { - } & \begin{array}{l}\text { Distanci } \\
\text { amiento } \\
\text { afectivo }\end{array} \\
\text { - } & \begin{array}{l}\text { Sentimi } \\
\text { entos de } \\
\text { frustraci } \\
\text { ón y } \\
\text { descont } \\
\text { ento. }\end{array} \\
\text { Depresi } \\
\text { ón. } \\
\text { Desespe } \\
\text { ranza. } \\
\text { Apatía. } \\
\text { Desilusi } \\
\text { ón. }\end{array}$ & $\begin{array}{ll}\text { - } & \text { Pérdida } \\
\text { de } & \text { signific } \\
\text { ado. } \\
\text { - } & \text { Pérdida } \\
\text { de } \\
\text { valores } \\
\text { - } \\
\text { Desapa } \\
\text { rición } \\
\text { de } \\
\text { expecta } \\
\text { tivas. } \\
\text { Modifi } \\
\text { cación } \\
\text { de } \\
\text { autoco } \\
\text { ncepto. } \\
\text { Desori } \\
\text { entació } \\
\text { n } \\
\text { cogniti } \\
\text { va. } \\
\text { Pérdida } \\
\text { de la }\end{array}$ & 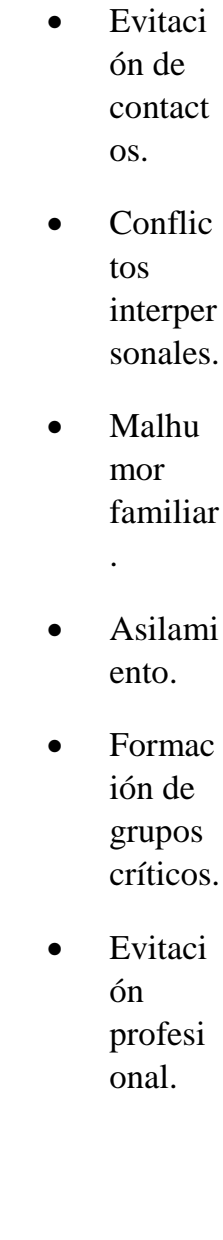 & $\begin{array}{ll}\text { - } & \text { Meno } \\
\mathrm{r} \\
\text { capac } \\
\text { idad } \\
\text { en el } \\
\text { trabaj } \\
\text { o o } \\
\text { bajo } \\
\text { rendi } \\
\text { mient } \\
\text { o. } \\
\text { Accio } \\
\text { nes } \\
\text { hostil } \\
\text { es. } \\
\text { Confl } \\
\text { ictos. } \\
\text { Falta } \\
\text { de } \\
\text { motiv } \\
\text { ación } \\
\text { por el } \\
\text { trabaj } \\
\text { o. }\end{array}$ \\
\hline
\end{tabular}




\begin{tabular}{|c|c|c|c|}
\hline $\begin{array}{l}\text { no } \\
\text { merm } \\
\text { a con } \\
\text { el } \\
\text { desca } \\
\text { nso. }\end{array}$ & $\begin{array}{ll} & \text { Sobre } \\
\text { implicaci } \\
\text { ón. }\end{array}$ & 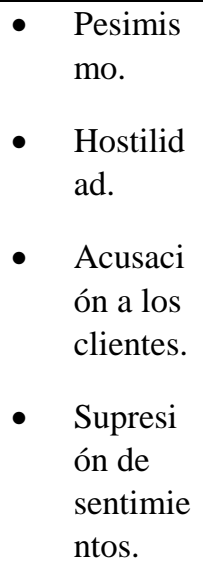 & $\begin{array}{ll} & \begin{array}{l}\text { creativi } \\
\text { dad. }\end{array} \\
\text { - } & \begin{array}{l}\text { Distrac } \\
\text { ción. }\end{array} \\
\text { - } & \text { Cinism } \\
\text { o. } \\
\text { - } \\
\text { Critisis } \\
\text { mo } \\
\text { general } \\
\text { izado. }\end{array}$ \\
\hline
\end{tabular}

\section{Antecedentes científicos}

Existen algunas investigaciones relacionadas con el desarrollo y la prevalencia del síndrome de burnout en docentes universitarios, y su asociación con diferentes variables de estudio. En el Ecuador no se ha encontrado información que permita ver la relación entre burnout y sus patologías.

(Pando Moreno, Aranda Beltran, Aldrete, Flores Salinas, \& Pozos Radillo, 2006), realizan el estudio de "Factores psicosociales y burnout en docentes del centro universitario de ciencias de la salud", aplicado a una muestra de 144 docentes con el objetivo de determinar la prevalencia específica de Burnout en docentes Universitarios y establecer cuáles factores psicosociales negativos del trabajo son los que se asocian a la presencia del Burnout en los docentes, encontrando que el 18,3\% están expuestos a factores psicosociales negativos y un $52,7 \%$ califican con síndrome de burnout, siendo las mujeres $(64,2 \%)$ quienes tienen las más altas tasas, y los solteros con $(61,7 \%)$.

(Quaas, 2006), realiza un estudio cuantitativo con perspectiva descriptiva relacional, en una muestra de 576 sujetos, para diagnosticar el síndrome de burnout en los docentes universitarios de la quinta región de Chile, así como identifica las técnicas de afrontamiento más utilizadas, determinando que el $72 \%$ no presenta burnout, mientras que del $28 \%$ restante, el $16 \%$ presenta un nivel reducido de burnout, un $10,2 \%$ presenta burnout moderado y sólo el 1,5\% tiene niveles altos de burnout.

(Ponce, Bulnes, Aliaga, Atalaya, \& Huertas, 2005), realizan su estudio descriptivo comparativo, para conocer la presencia del síndrome del quemado por estrés laboral asistencial en grupos de docentes universitarios, pertenecientes a distintas áreas de especialidad, con una muestras de 274 docentes, determinando que el $46.3 \%$ de los docentes tienen burnout en niveles significativos; el $7.3 \%$ se encuentran en la fase intermedia y próximos a avanzar a la fase final; $y$ un $46,4 \%$ se encuentran en una fase inicial cuyo desarrollo es lento.

(Arquero \& Donoso, 2006), realizan un estudio para medir la incidencia del síndrome de quemarse en el trabajo en una muestra piloto de docentes universitarios de contabilidad, utiliza como instrumento el MBI estándar, obteniendo como resultados que el $28 \%$ de la muestra tiene niveles significativamente altos en dos o más de las escalas y el 14\% presenta en las tres escalas. 
(Avargues, Borda, \& López, 2010), Estudian el core of burnout y los síntomas de estrés en el personal de Universidad. Prevalencia e influencia de variables de carácter sociodemográfico y laboral, de una población inicial de 315 personas, se evidencia que el 29,2\% tiene niveles de cansancio emocional alto, el 17,5\% tiene niveles de despersonalización alto, y el $32,7 \%$ padece de sintomatología de estrés aguda.

(Ruiz de Chávez, Pando Moreno, Aranda Beltran, \& Almeida Perales, 2014), estudia la relación que existe entre el síndrome de Burnout y el Work Engagement, con variables demográficas y laborales, en docentes universitarios de Zacatecas (México) a través de un estudio observacional, analítico, transversal, en una muestra de 156 docentes, obteniendo una prevalencia del síndrome de burnout del 63,5\%, y por dimensiones, la siguiente distribución: 47,4\% con agotamiento emocional, 26,9\% con despersonalización y el 76,9\% mostró bajos niveles de realización personal en el trabajo.

(Moreno, Garroza, \& Rodríguez, 2009), realizan su estudio sobre el burnout del profesorado universitario y las intenciones de abandono, para analizar los posibles efectos de mediación del burnout en la relación entre demandas, conflicto interpersonal e intenciones de abandono, con un estudio de corte transversal y de muestreo aleatorio, obteniéndose como resultado que tanto el agotamiento emocional como el distanciamiento mediaron los efectos que las demandas y el conflicto tienen sobre las intenciones de abandono, mientras que los recursos laborales predijeron negativamente las intenciones de abandono de manera directa. Los resultados obtenidos apoyaron la aplicabilidad de un modelo extendido de demandas-recursos laborales (JD-R model) en profesores universitarios.

(Ferrel, Pedraza, \& Rubio, 2010), realizan un estudio para establecer y describir la presencia o ausencia del síndrome del quemado (burnout) en los docentes universitarios, en una muestra de 225 docentes, encontraron una fuerte presencia de desgaste emocional, un bajo nivel de despersonalización, y una presencia menor de realización personal.

(Pando-Moreno, 2006), realizó un estudio transversal descriptivo en una muestra de 565 docentes, para determinar la presencia de los factores psicosociales laborales negativos percibidos por el trabajador y su relación con el síndrome de burnout, en docentes de la Universidad del Valle de Atemajac, en Guadalajara, México.

(Vélez, 2013), realiza un estudio descriptivo, correlacional de corte transversal, en una población de 90 docentes, para determinar la relación entre factores psicosociales y el síndrome de burnout, encontrando que el 42,2\% de la población puede considerarse con síndrome de burnout.

\section{Pregunta de investigación.}

¿Cuál es la presencia del síndrome de burnout en docentes universitarios en Latinoamérica y España? 
¿Qué estudios en América latina y España, existen sobre el síndrome de burnout en docentes universitarios?

\section{Objetivos}

El presente estudio pretende analizar la presencia del síndrome de burnout en docentes universitarios en Latinoamérica y España, como un elemento importante en el bienestar y en la calidad de vida laboral del docente.

\section{Objetivos específicos}

1. Buscar información de estudios realizados sobre el síndrome de burnout en docentes universitarios en Latinoamérica y España, en las bases de datos de la UTE.

2. Conocer qué países han realizado su aporte científico en este campo.

3. Determinar cuál es el instrumento más utilizado para los estudios de burnout.

4. Determinar los niveles de burnout en sus tres componentes, así como la presencia del síndrome.

\section{Materiales y métodos}

La presente investigación es de tipo bibliográfica, descriptiva y analítica, donde se utilizaron 35 documentos empíricos (artículos científicos) de los diez últimos años, referentes al tema, obtenidos de las siguientes bases científicas: Ebsco, e-libro; así como en repositorios como Dialnet, SciELO, Redalyc y Google académico; realizándose la última búsqueda el 30 de octubre de 2015.

El proceso de revisión bibliográfica se realizará en los siguientes tres pasos:

1. Fuente de la investigación bibliográfica:

a. Tipo de fuente.

b. Tipo de documento implicado.

c. Tipo de edición que avala el documento.

2. Proceso de análisis de la información.

3. Resultados de la revisión bibliográfica.

\section{Resultados}

La evolución de la producción bibliográfica sobre el burnout en docentes universitarios, realizada entre los años 2005 a 2015, de los 35 artículos empíricos seleccionados, se evidencia que la mayoría de estudios se han realizado en Colombia (28,6\%), México (22,9\%) y España (20,0\%), no se encontraron estudios en Uruguay, Paraguay, Bolivia, Ecuador, Panamá, y otros países centroamericanos. En relación a la población de estudio, existe una mayor participación de hombres $3533(51,6 \%)$ que de mujeres 2792 (40,8\%) de una población total de 6842 
participantes. Existieron 4 estudios que no presentaron la población de estudio dividido en hombres y mujeres, que representa $517(7,6 \%)$ personas (Tabla 1).

Tabla 1. Países y población de estudio, donde se han realizado estudios en Latinoamérica durante el período 2005-2015.

\begin{tabular}{|c|c|c|c|c|c|c|c|c|}
\hline \multirow[t]{3}{*}{ País } & \multicolumn{2}{|c|}{ Número De Documentos } & \multicolumn{6}{|c|}{ Población De Estudio } \\
\hline & \multirow[b]{2}{*}{$\mathbf{N}$} & \multirow[b]{2}{*}{$(\%)$} & \multicolumn{2}{|c|}{ Hombres } & \multicolumn{2}{|c|}{ Mujeres } & \multicolumn{2}{|l|}{ Total } \\
\hline & & & $\mathbf{N}$ & $\%$ & $\mathbf{N}$ & $\%$ & $\mathbf{N}$ & $\%$ \\
\hline Brasil & 2 & 5,7 & 99 & 2,8 & 70 & 2,5 & 198 & 2,9 \\
\hline Colombia & 10 & 28,6 & 443 & 12,5 & 434 & 15.5 & 1246 & 18,2 \\
\hline Chile & 2 & 5,7 & 387 & 11,0 & 189 & 6,8 & 695 & 10.2 \\
\hline Perú & 2 & 5,7 & 245 & 6,9 & 90 & 3,2 & 335 & 4,9 \\
\hline Venezuela & 1 & 2,9 & 61 & 1,7 & 84 & 3,0 & 145 & 2,1 \\
\hline México & 8 & 22,9 & 1150 & 32,6 & 856 & 30,7 & 2006 & 29,3 \\
\hline España & 7 & 20,0 & 1017 & 28,8 & 910 & 32,6 & 1927 & 28,2 \\
\hline Nicaragua & 1 & 2,9 & 48 & 1,4 & 61 & 2,2 & 109 & 1,6 \\
\hline República Dominicana & 1 & 2,9 & 34 & 1,0 & 27 & 1,0 & 61 & 0,9 \\
\hline Argentina & 1 & 2,9 & 49 & 1,4 & 71 & 2,5 & 120 & 1,8 \\
\hline Total & 35 & 100 & 3533 & 51,6 & 2792 & 40,8 & 6842 & 100 \\
\hline
\end{tabular}

En la Tabla 2, se aprecia que los años con mayor cantidad de estudios realizados son el 2009 y 2013 con $6(17,1 \%)$ estudios, y los años 2006 y 2010 con $5(14,3 \%)$ estudios.

Tabla 2.Producción científica por años: período 2005-2015.

\begin{tabular}{lll} 
Año & Número De Documentos & $\begin{array}{l}\text { Porcentaje } \\
(\%)\end{array}$ \\
\hline Año 2005 & 1 & 2,9 \\
Año 2006 & 5 & 14,3 \\
Año 2007 & 1 & 2,9 \\
Año 2008 & 3 & 8,6 \\
Año 2009 & 6 & 17,1 \\
Año 2010 & 5 & 14,3 \\
Año 2011 & 3 & 8,6 \\
Año 2012 & 2 & 5,7 \\
\hline
\end{tabular}




\begin{tabular}{lll}
\hline Año 2013 & 6 & 17,1 \\
Año 2014 & 3 & 8,6 \\
Año 2015 & 0 & 0,0 \\
Total & $\mathbf{3 5}$ & $\mathbf{1 0 0 , 0}$ \\
\hline
\end{tabular}

La Tabla 3, muestra que el cuestionario más utilizado en los estudios de burnout, en docentes universitarios es el Maslach Burnout Inventory (MBI) con el 85,7\%, mientras que los otros cuestionarios como el CBB (2,9\%), CBP-R (5,7\%) y el CESQT (5,7\%), son aún poco utilizados.

Tabla 3. Instrumentos de evaluación más citados. Tests más utilizados en la investigación sobre burnout en docentes universitarios a lo largo del período 2005-2015.

\begin{tabular}{|c|c|c|}
\hline Cuestionario & Número De Documentos & $\begin{array}{l}\text { Porcentaje } \\
(\%)\end{array}$ \\
\hline $\mathrm{Mbi}^{*}$ & 30 & $85,7 \%$ \\
\hline $\mathrm{Cbb}^{* *}$ & 1 & $2,9 \%$ \\
\hline Cbp- $\mathrm{R}^{* * * *}$ & 2 & $5,7 \%$ \\
\hline Cesqt $^{* * * *}$ & 2 & $5,7 \%$ \\
\hline Total & 35 & $100,0 \%$ \\
\hline \multicolumn{3}{|c|}{$\begin{array}{c}* \mathrm{MBI}=\text { Maslach Burnout Inventory } \\
* * \mathrm{CBB}=\text { Cuestionario Breve de Burnout } \\
* * * \mathrm{CBP}-\mathrm{R}=\text { Cuestionario de Burnout del Profesorado }\end{array}$} \\
\hline
\end{tabular}

La Tabla 4, muestra las tres escalas de Burnout, en la escala del Agotamiento emocional (AE) se establece que el 966 (22,2\%) sujetos de la muestra tiene un valor alto, siendo México $(6,8 \%)$, España $(6,4 \%)$ y Colombia $(4,2 \%)$ los países con los valores más tos de la escala. En la escala de Despersonalización (DP) se tienen 753 (17,4\%) tienen un valor alto, donde destacan España (6,7\%) y Colombia (3,6\%). En la escala de realización personal (RP), 2351 (54,7\%) sujetos tienen puntuación baja, siendo México con el 25,5\% el país más representativo, seguido de Colombia con el 17,3\% y España con el 6,3\%.

Tabla 4. Escalas de Burnout.

\begin{tabular}{|c|c|c|c|c|c|c|c|c|c|c|c|c|c|c|c|c|c|c|}
\hline & \multicolumn{5}{|c|}{ Agotamiento Emocional, Ae } & \multicolumn{6}{|c|}{ Despersonalización, Dp } & & \multicolumn{6}{|c|}{ Realización Personal, Rp } \\
\hline & Bajo & \multicolumn{2}{|c|}{${ }^{\text {Medi }}$} & \multicolumn{2}{|c|}{ Alto } & \multicolumn{2}{|c|}{ Bajo } & \multicolumn{2}{|c|}{ o $^{\text {Medi }}$} & \multicolumn{2}{|c|}{ Alto } & \multicolumn{3}{|c|}{ Bajo } & \multicolumn{2}{|c|}{${ }^{\text {Medi }}$} & \multicolumn{2}{|c|}{ Alto } \\
\hline País & $\mathbf{N}$ & $\mathbf{N}$ & $\%$ & $\mathbf{N}$ & & $\mathbf{N}$ & $\%$ & $\mathbf{N}$ & & $\mathbf{N}$ & & & $\mathbf{N}$ & $\%$ & $\mathbf{N}$ & $\%$ & $\mathbf{N}$ & $\%$ \\
\hline Brasil & 1.4 & 63 & $\begin{array}{l}1 . \\
5\end{array}$ & 68 & $\begin{array}{l}1 . \\
6\end{array}$ & 76 & 1.8 & 74 & $\begin{array}{l}1 . \\
7\end{array}$ & 40 & $\begin{array}{l}0 . \\
9\end{array}$ & & 0 & 2.1 & 59 & $\begin{array}{l}1 . \\
4\end{array}$ & 43 & $\begin{array}{l}1 . \\
0\end{array}$ \\
\hline
\end{tabular}




\begin{tabular}{|c|c|c|c|c|c|c|c|c|c|c|c|c|c|c|c|c|c|c|}
\hline Colombia & $\begin{array}{l}64 \\
9\end{array}$ & $\begin{array}{l}15 . \\
0\end{array}$ & $\begin{array}{l}37 \\
8\end{array}$ & $\begin{array}{l}8 . \\
7\end{array}$ & $\begin{array}{l}18 \\
2\end{array}$ & $\begin{array}{l}4 . \\
2\end{array}$ & $\begin{array}{l}66 \\
3\end{array}$ & $\begin{array}{l}15 . \\
4\end{array}$ & $\begin{array}{l}38 \\
8\end{array}$ & $\begin{array}{l}9 . \\
0\end{array}$ & $\begin{array}{l}15 \\
5\end{array}$ & $\begin{array}{l}3 . \\
6\end{array}$ & $\begin{array}{l}74 \\
0\end{array}$ & $\begin{array}{l}17 . \\
3\end{array}$ & $\begin{array}{l}27 \\
2\end{array}$ & $\begin{array}{l}6 . \\
4\end{array}$ & $\begin{array}{l}20 \\
0\end{array}$ & $\begin{array}{l}4 . \\
7\end{array}$ \\
\hline Chile & 70 & 1.6 & 30 & $\begin{array}{l}0 . \\
7\end{array}$ & 18 & $\begin{array}{l}0 . \\
4\end{array}$ & 32 & 0.7 & 51 & $\begin{array}{l}1 . \\
2\end{array}$ & 30 & $\begin{array}{l}0 . \\
7\end{array}$ & 11 & 0.3 & 93 & $\begin{array}{l}2 . \\
2\end{array}$ & 14 & $\begin{array}{l}0 . \\
3\end{array}$ \\
\hline Perú & 53 & 1.2 & 4 & $\begin{array}{l}0 . \\
1\end{array}$ & 4 & $\begin{array}{l}0 . \\
1\end{array}$ & 36 & 0.8 & 19 & $\begin{array}{l}0 . \\
4\end{array}$ & 6 & $\begin{array}{l}0 . \\
1\end{array}$ & 10 & 0.2 & 20 & $\begin{array}{l}0 . \\
5\end{array}$ & 31 & $\begin{array}{l}0 . \\
7\end{array}$ \\
\hline Venezuela & 43 & 1.0 & 49 & $\begin{array}{l}1 . \\
1\end{array}$ & 50 & $\begin{array}{l}1 . \\
2\end{array}$ & 94 & 2.2 & 30 & $\begin{array}{l}0 . \\
7\end{array}$ & 18 & $\begin{array}{l}0 . \\
4\end{array}$ & 85 & 2.0 & 12 & $\begin{array}{l}0 . \\
3\end{array}$ & 45 & $\begin{array}{l}1 . \\
1\end{array}$ \\
\hline México & $\begin{array}{l}10 \\
97\end{array}$ & $\begin{array}{r}2 \\
5.3\end{array}$ & $\begin{array}{r}3 \\
22\end{array}$ & $\begin{array}{l}7 \\
.4\end{array}$ & $\begin{array}{r}2 \\
94\end{array}$ & ${ }^{6}{ }^{6}$ & $\begin{array}{l}1 \\
36 \\
2\end{array}$ & $\begin{array}{r}3 \\
1.6\end{array}$ & $\begin{array}{r}1 \\
97\end{array}$ & ${ }^{4}$ & $\begin{array}{r}1 \\
19\end{array}$ &.$^{2}$ & $\begin{array}{l}1 \\
09 \\
1\end{array}$ & $\begin{array}{r}2 \\
5.5\end{array}$ & $\begin{array}{r}2 \\
94\end{array}$ & $\begin{array}{c}6 \\
.9\end{array}$ & $\begin{array}{r}2 \\
67\end{array}$ &.${ }^{6}$ \\
\hline España & $\begin{array}{l}17 \\
3\end{array}$ & 4.0 & $\begin{array}{l}17 \\
3\end{array}$ & $\begin{array}{l}4 . \\
0\end{array}$ & $\begin{array}{l}27 \\
6\end{array}$ & $\begin{array}{l}6 . \\
4\end{array}$ & $\begin{array}{l}17 \\
8\end{array}$ & 4.1 & $\begin{array}{l}18 \\
8\end{array}$ & $\begin{array}{l}4 . \\
3\end{array}$ & $\begin{array}{l}29 \\
2\end{array}$ & $\begin{array}{l}6 . \\
7\end{array}$ & $\begin{array}{l}26 \\
9\end{array}$ & 6.3 & $\begin{array}{l}20 \\
0\end{array}$ & $\begin{array}{l}4 . \\
7\end{array}$ & $\begin{array}{l}16 \\
8\end{array}$ & $\begin{array}{l}3 . \\
9\end{array}$ \\
\hline Nicaragua & 99 & 2.3 & 4 & $\begin{array}{l}0 . \\
1\end{array}$ & 5 & $\begin{array}{l}0 . \\
1\end{array}$ & 11 & 0.3 & 67 & $\begin{array}{l}1 . \\
6\end{array}$ & 31 & $\begin{array}{l}0 . \\
7\end{array}$ & 13 & 0.3 & 33 & $\begin{array}{l}0 . \\
8\end{array}$ & 62 & $\begin{array}{l}1 . \\
5\end{array}$ \\
\hline $\begin{array}{l}\text { República } \\
\text { Dominicana }\end{array}$ & 50 & 1.2 & 5 & $\begin{array}{l}0 . \\
1\end{array}$ & 5 & $\begin{array}{l}0 . \\
1\end{array}$ & 45 & 1.0 & 10 & $\begin{array}{l}0 . \\
2\end{array}$ & 4 & $\begin{array}{l}0 . \\
1\end{array}$ & 5 & 0.1 & 10 & $\begin{array}{l}0 . \\
2\end{array}$ & 45 & $\begin{array}{l}1 . \\
1\end{array}$ \\
\hline Argentina & 22 & 0.5 & 32 & $\begin{array}{l}0 . \\
7\end{array}$ & 64 & $\begin{array}{l}1 . \\
5\end{array}$ & 21 & 0.5 & 39 & $\begin{array}{l}0 . \\
9\end{array}$ & 58 & $\begin{array}{l}1 . \\
3\end{array}$ & 37 & 0.9 & 44 & $\begin{array}{l}1 . \\
0\end{array}$ & 37 & $\begin{array}{l}0 . \\
9\end{array}$ \\
\hline TOTAL & $\begin{array}{l}23 \\
17\end{array}$ & $\begin{array}{l}53 . \\
5\end{array}$ & $\begin{array}{l}10 \\
60\end{array}$ & $\begin{array}{l}24 \\
.5\end{array}$ & $\begin{array}{l}96 \\
6\end{array}$ & $\begin{array}{l}22 \\
.2\end{array}$ & $\begin{array}{l}25 \\
18\end{array}$ & $\begin{array}{l}58 . \\
4\end{array}$ & $\begin{array}{l}10 \\
63\end{array}$ & $\begin{array}{l}24 \\
.5\end{array}$ & $\begin{array}{l}75 \\
3\end{array}$ & $\begin{array}{l}17 \\
.4\end{array}$ & $\begin{array}{l}23 \\
51\end{array}$ & $\begin{array}{l}54 . \\
7\end{array}$ & $\begin{array}{l}10 \\
37\end{array}$ & $\begin{array}{l}24 \\
.1\end{array}$ & $\begin{array}{l}91 \\
2\end{array}$ & $\begin{array}{l}21 \\
.3\end{array}$ \\
\hline
\end{tabular}

En lo referente a la presencia de Burnout (Tabla 5), las puntuaciones más altas las tiene México (7,3\%), Colombia (5,2\%) y España (4,1\%); y en general el 19,8\% de la muestra de docentes universitarios analizada, tiene alta presencia de Burnout, el 15,4\% tiene una valor medio y el 28,0\% presenta un bajo nivel de Burnout.

Tabla 5. Presencia de burnout.

\begin{tabular}{lllllllll}
\hline & \multicolumn{3}{c}{ Presencia De Burnout } & & \\
& & Bajo & & Medio & & Alto & \\
\hline Pais & Población & $\mathbf{N}$ & $\mathbf{\%}$ & $\mathbf{N}$ & $\mathbf{\%}$ & $\mathbf{N}$ & \% \\
Brasil & 198 & 60 & 0.9 & 65 & 1.0 & 66 & 1.0 \\
Colombia & 1246 & 504 & 7.4 & 346 & 5.1 & 359 & 5.2 \\
Chile & 695 & 39 & 0.6 & 58 & 0.8 & 20 & 0.3 \\
Perú & 335 & 40 & 0.6 & 14 & 0.2 & 7 & 0.1 \\
Venezuela & 145 & 61 & 0.9 & 30 & 0.4 & 51 & 0.7 \\
México & 2006 & 909 & 13.3 & 271 & 4.0 & 501 & 7.3 \\
España & 1927 & 173 & 2.5 & 187 & 2.7 & 279 & 4.1 \\
Nicaragua & 109 & 57 & 0.8 & 35 & 0.5 & 16 & 0.2 \\
República Dominicana & 61 & 47 & 0.7 & 8 & 0.1 & 5 & 0.1 \\
Argentina & 120 & 27 & 0.4 & 38 & 0.6 & 53 & 0.8 \\
\hline
\end{tabular}




$\begin{array}{llllllll}\text { TOTAL } & 6842 & 1916 & 28.0 & 1053 & 15.4 & 1357 & 19.8\end{array}$

\section{Discusión}

Las características que definen al síndrome de Burnout son: agotamiento emocional, despersonalización y la baja realización personal. Se realizó una revisión bibliográfica sobre el síndrome de Burnout en docentes universitarios, desde el 2005 hasta el 30 de octubre de 2015, encontrándose que Colombia, es el país de habla hispana que más aportes ha realizado 10 $(28,6 \%)$ documentos.

Existe mayor participación de hombres 3533 (51,6\%) que de mujeres 2792 (40,8\%) de una población total de 6842 sujetos, siendo México el país que presenta la mayor muestra poblacional, $2006(29,3 \%)$.

El cuestionario MBI propuesto por Maslach \& Jackson $(1981,1986)$ es el más utilizado $(85,7 \%)$. Su alta aplicación, se debe a alta fiabilidad y alta validez sobre las tres dimensiones que componen el método, y es el cuestionario más utilizado internacionalmente (Martínez Pérez, 2010).

En una revisión de las escalas realizada en el año 1986, alcanzaron valores de fiabilidad de Cronbach aceptables, con una consistencia interna de 0.80 y la validez convergente y la discriminante han sido adecuadas. Respecto a la fiabilidad por factores se obtuvieron valores de 0.90 para el agotamiento personal, 0.79 para la despersonalización y 0.71 para la baja realización personal. (Martínez Pérez, 2010)

Adicionalmente, (Gil-Monte \& Peiró, 1999), en su estudio sobre la validez factorial del Maslach Burnout Inventoy en una muestra multiocupacional, concluye que la adaptación al castellano del MBI reúne los suficientes requisitos de validez factorial y consistencia interna como para ser empleada en la estimación del síndrome de quemarse por el trabajo en nuestro contexto sociocultural.

En lo referente a las escalas de Burnout, México tiene el mayor valor en Agotamiento Emocional (6,8\%), España (6,7\%) tiene el mayor puntaje en la escala de despersonalización, y México $(25,5)$ tiene el nivel más bajo en la escala de realización personal. Finalmente, México es el país con mayor porcentaje alto en la presencia de Burnout $(7,3 \%)$.

\section{Conclusiones}

Del estudio realizado en base a los datos de los 35 documentos seleccionados, se concluye que los países que más altos niveles de burnout en docentes universitarios presentan, son México, Colombia y España. No todos los documentos encontrados, tienen uniformidad en la presentación y análisis de los resultados. 


\section{Bibliografía}

Arquero, J., \& Donoso, A. (2006). El impacto del síndrome burnout en los profesores noveles. Un estudio piloto. Revista de enseñanza universitaria, $N^{\circ} 27 ., 69-82$.

Avargues, M. B. (2010). Estrés laboral y síndrome de burnout en la Universidad: análisis descriptivo de la situación actual y revisión de las principales líneas de investigación. Anuario de Psicología Clínica y de la Salud., 73-78.

Avargues, M. L., Borda, M., \& López, A. M. (2010). El core of burnout y los síntomas de estrés en el personal de Universidad. Prevalencia e influencia de variables de carácter sociodemográfico y laboral. Boletín de Psicología, No. 99, Julio 2010., 89-101.

Bermejo, T. L., \& Prieto, U. M. (2005). Malestar docente y creencias de autoeficacia del profesor. Revista española de pedagogía, año LXIII, $n .^{\circ} 232$, septiembre-diciembre 2005., 493-510.

Berrio, N. M. (2011). Estrés académico. Revista de psicología. Universidad de Antioquia. Vol. 3. N²., 6582.

Bestatén, M. (2008). El trabajo y tu salud. Madrid: INSHT.

Castro, R. (2005). Sindrome de Burnout o desgaste profesional. ANUARIO N ${ }^{\circ} 28,158-184$.

De la Torre, R. C. (29 de Octubre de 2006). El malestar docente: Un femómeno de relevancia internacional. Obtenido de stellae.usc.es: http://stellae.usc.es/red/file/download/5625

Dejours, C. (1998). De la psicopatología a la psicodinámica del trabajo. En D. Dessors, \& M. Guiho-Bailly, Organizacion del trabajo y salud. (pág. 24). Buenos ASires: Ed. Lumen Hvmanitas.

Durán, D. M., \& Extremera, P. N. (2001). Burnout en profesionales de la enseñanza: Un estudio en educación primaria, secundaria y superior. Psicfología del trabajo y de las organizaciones., 45-62.

Esteve, J. M. (1987). El malestar docente. Barcelona.

Ferrel, R., Pedraza, C., \& Rubio, B. (2010). El síndrome de quemarse en el trabajo (burnout) en docentes universitarios. Duazary, 15-28.

Gil-Monte, P. R., \& Peiró, J. M. (1999). Validez Factorial Del Maslach Burnout Inventory En Una Muestra Multiocupacional. Psicothema, 1999. Vol. 11, $n^{o} 3 .$, pp. 679-689.

Guerrero Barona, E. (2003). Análisis pormenorizado de los grados de burnout y técnicas de afrontamiento del estrés docente en profesorado universitario. Anales de psicología., 145-158.

Guerrero, E. (2001). Una investigación con docentes universitarios sobre el afrontamiento del estrés laboral y el síndrome del "quemado". Revista Iberoamericana de Educación, 1-22.

Ibañez, E. A. (2012). Prevalencia y factores asociados al Síndrome de burnout en docentes de odontología. Fundación Universitaria San Martín, año 2011. Revista Colombiana de Enfermería, Vol. 7, $N^{\circ} 7$. , 105-111.

Leka, S. (2004). La organización del trabajo y el estres. Ginebra: OMS. 
Marín, H., \& Paredes, M. (2002). Estudio del síndrome de burnout o desgaste profesional en los profesores de la Universidad de los Andes. Educere, Investigación, Año 6, $N^{\circ} 17 ., 29-36$.

Marrau, C. (2004). El síndrome de Burnout y sus posibles consecuencias en el trabajador docente. Fundamentos de humanidades., 53-68.

Martínez Pérez, A. (2010). El Síndrome De Burnout. Evolución Conceptual Y Estado Actual De La Cuestión. Vivat Academia. $n^{\circ}$ 112. Septiembre. 2010., 1-40.

Minteguiaga, A. P. (2013). El papel estratégico del personal académico en la transformación de la educación superior en el Ecuador. Cuaderno de Política Pública., 1-58.

Moreno, B. G. (2001). Desgadte profesional (burnout), personalidad y salud percibida. Empleo, estrés y salud, 59-83.

Moreno, J. B., Garroza, H. E., \& Rodríguez, C. R. (2009). El burnout del profesorado universitario y las intenciones de abandono. Un estudio multi-muestra. Revista de Psocilogía del Trabajo y de las Organizaciones. Vol. 25, $N^{\circ} 2 ., 149-163$.

Orgambídez Alejandro, P. P. (2015). Estrés de rol y satisfacción laboral: examinando el papel mediador del engagement en el trabajo. Journal of Work and Organizational Psychology, 69-77.

Pando Moreno, M., Aranda Beltran, C., Aldrete, M., Flores Salinas, E., \& Pozos Radillo, E. (2006). Factores psicosociales y burnout en docentes del centro universitario de ciencias de la salud. Investigación en salud. Vol. VIII., 173-177.

Pando-Moreno, M. C.-T.-G.-M.-d. (2006). Factores psicosociales y síndrome de burnout en docentes de la universidad del valle de Atemajac, Guadalajara, México. Salud en Tabasco, vol. 1, núm., 523-529.

Ponce, C., Bulnes, M., Aliaga, J., Atalaya, M., \& Huertas, R. (2005). El Síndrome del "Quemado" por estrés laboral asistencial en grupos de docentes universitarios. IIPSI., 87-112.

Quaas, C. (2006). Diagnóstico de burnout y técnicas de afrontamiento al estrés en profesores universitarios de la Quinta Región de Chile. Psicoperspectivas., 65-75.

Quiceno, J. M., \& Alpi, S. V. (2007). Burnout: "Síndrome de quemarse en el trabajo (SQT)”. Acta Colombiana de Psicología 10 (2)., 117-125.

Ruiz de Chávez, D., Pando Moreno, M., Aranda Beltran, C., \& Almeida Perales, C. (2014). Burnout y Work Engagement en docentes Universitarios de Zacatecas. Cienciaytrabajo, Año 16, $N^{\circ}$ 50., 116-120.

Tifner, S. M. (2006). Burnout en el colectivo docente. Revista de Humanidades, 12., 279-291.

Vélez, P. M. (2013). Relación de los factores de riesgo psicosociales y el síndrome de burnout en docentes de planta de la Universidad Francisco de Pasula Santander, en el período de 2011-2012. Ciencia y Cuidad, Vol. 10, $N^{\circ}$ 2, 85-95.

Zabala, J. (2008). Estrés y burnout docente: conceptos, causas y efectos. Educación Vol. XVII, N 32, 6786. 